\title{
Nuove opzioni terapeutiche per la malattia renale policistica
}

\author{
Domenico Santoro, Vincenzo Pellicanò, Luca Visconti, Viviana Lacava, CarloAlberto Ricciardi, Antonio Lacquaniti, \\ Valeria Cernaro, Michele Buemi
}

Dipartimento di Medicina Clinica e Sperimentale, Università di Messina, Messina

\begin{abstract}
New options for the management of polycystic kidney disease
Autosomal dominant polycystic kidney disease (ADPKD) is the most common renal genetic disorder being responsible for $\sim 10 \%$ of cases of end-stage renal disease. At present, ADPKD therapy is essentially supportive, limited to blood pressure reduction and to symptomatic treatment of disease complications. However, recent findings on the pathophysiology of the disease have stimulated the research on new therapeutic strategies in an attempt to stop ADPKD progression. Mammalian target of rapamycin (mTOR) inhibitors, sirolimus and everolimus, seem to have some effects in ADPKD patients, but the therapeutic role of these drugs remains uncertain. The main candidates for the treatment of renal and hepatic cysts are the somatostatin analogues lanreotide and octreotide. The most promising therapy for the treatment of progressive ADPKD is the Vasopressin receptor (V2) antagonist tolvaptan. Other therapeutic strategies are currently under investigation but data are still not sufficient to establish if these approaches may provide consistent benefits in decelerating the progression of ADPKD in the next future.
\end{abstract}

Keywords: Analoghi delle somatostatine, AMPc, m-TOR, PKD, Tolvaptan

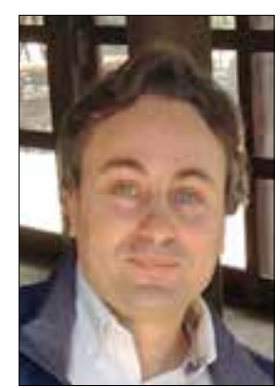

Domenico Santoro

\section{Introduzione}

La malattia del rene policistico (PKD) è la malattia genetica renale più comune, essendo responsabile di circa il $10 \%$ dei casi di malattia renale allo stadio terminale (ESRD) (1). Ne esistono due forme principali, una a trasmissione autosomica recessiva (ARPKD), molto più rara, e un'altra a trasmissione autosomica dominante (ADPKD). In quest'ultima, le mutazioni sono state riscontrate sul gene "PKD1" (circa l' $85 \%$ dei casi) e sul gene "PKD 2" (il restante $15 \%$ dei casi), codificanti rispettivamente per le proteine

Accepted: May 2, 2016

Published online: May 30, 2016

Indirizzo per la corrispondenza:

Prof. Domenico Santoro

AOU G. Martino PAD C

Dipartimento di Medicina Clinica e Sperimentale

Università di Messina

Via Consolare Valeria

98100 Messina

santisi@hotmail.com policistina 1 e policistina 2 (2). Un terzo gene "PKD3" era stato ipotizzato in passato per alcune forme di malattia policistica, non-PKD1 e non-PKD2, ma non è stato mai identificato.

L'interazione reciproca delle due policistine porta alla formazione di un complesso di segnalazione multifunzionale coinvolto nella regolazione del $\mathrm{Ca} 2$ + intracellulare e nei processi di sviluppo e di rigenerazione epiteliale (Fig. 1). Essa svolge, inoltre, un ruolo chiave nel mantenimento di un fenotipo differenziato delle cellule epiteliali del rene. Di conseguenza, la produzione di policistine alterate o mutate, come avviene nella patologia cistica, trasformerebbe le cellule tubulo-epiteliali renali in cellule poco differenziate, che proliferano in maniera aberrante formando strutture cistiche (1). Un altro importante fattore patogenetico è, probabilmente, rappresentato dalle anomalie delle ciglia primarie, strutture che trovano residenza sulla superficie apicale delle cellule epiteliali del tubulo renale. Questi organelli intervengono fisiologicamente nella regolazione della polarità planare delle cellule e nella proliferazione e nella differenziazione cellulari. Inoltre, i prodotti genici di PKD1 e PKD2 contribuiscono alla struttura e alla funzione corretta delle ciglia e, infatti, le mutazioni genetiche che causano difetti del ciglio primario sono state associate alla PKD (3).

La storia naturale della malattia prevede la sostituzione progressiva della normale struttura parenchimale del rene con numerose cisti a contenuto liquido che aumentano 


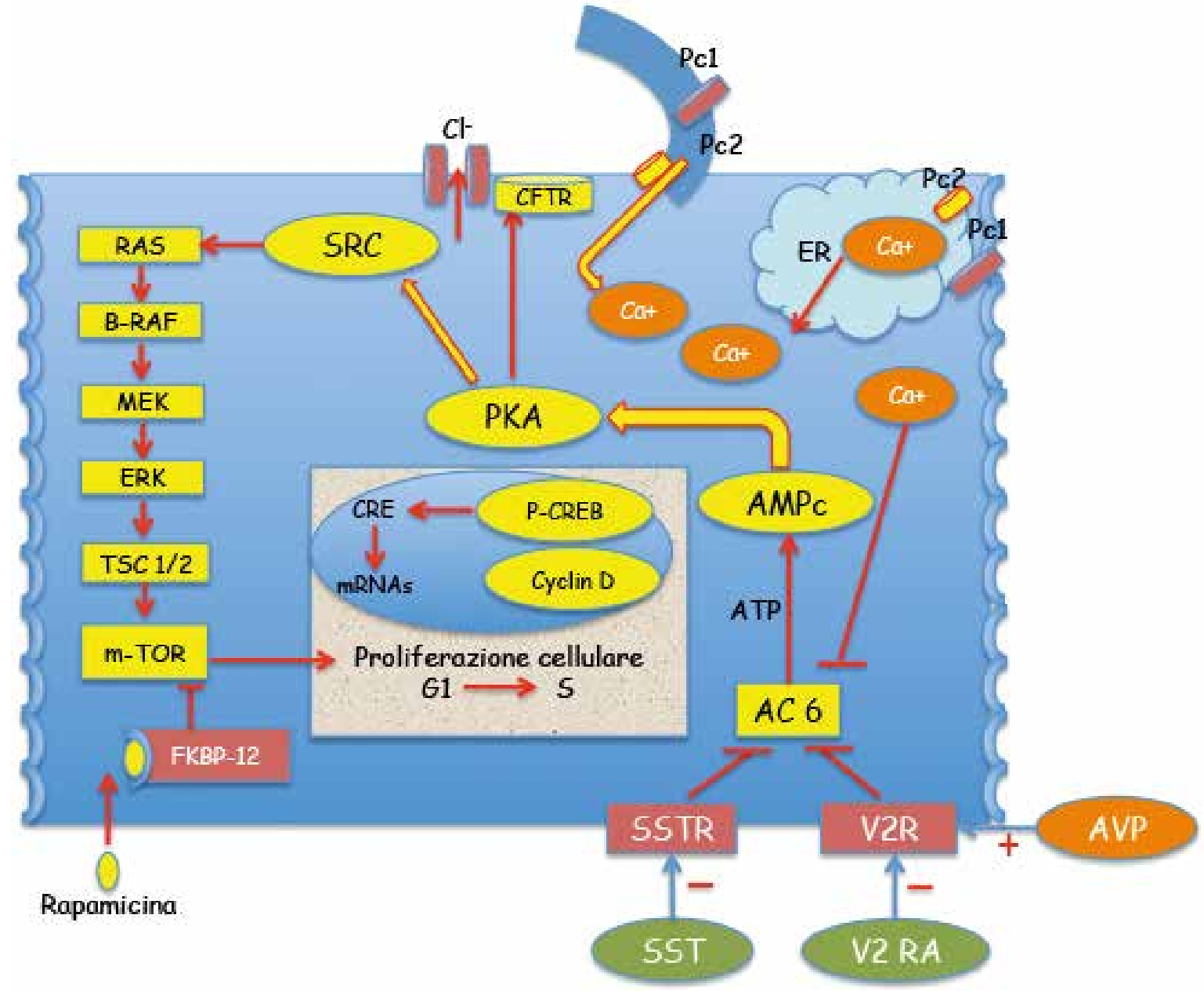

Fig. 1 - Cellula tubulare: meccanismi cellulari coinvolti nella fisiopatologia del rene policistico e corrispettivi target terapeutici (66). Meccanismi cellulari alterati. L'aumentata concentrazione di AMPc è secondaria a differenti meccanismi: riduzione del Calcio che normalmente inibisce AC-6, incrementati livelli circolanti di AVP, upregolazione di AVP V2R. AMPc causa un'iperattivazione delle PKA che stimolano la proliferazione cellulare con attivazione delle PCREB/Cyclin D all'interno del nucleo e successiva proliferazione cellulare attraverso la cascata SRC e RAS. Quest'ultimo meccanismo determina l'attivazione di ERK e TSC1/2, che porta alla stimolazione di mTOR con la conseguente progressione della proliferazione cellulare da G1 a S. In aggiunta, l'aumentata concentrazione dei livelli di AMPc contribuisce alla crescita delle cisti attraverso una secrezione di fluidi e di cloro.

Target terapeutici. 1) II legame della somatostatina al suo recettore inibisce l'attivazione di AC-6, determinando una riduzione della concentrazione di AMPc. 2) I vaptani sono degli antagonisti non peptidici della vasopressina; spiazzano il legame della vasopressina con il suo recettore (V2R), determinando una riduzione dei livelli di AMPc. 3) La rapamicina è un inibitore diretto di mTOR attraverso la sua interazione con FKBP-12 all'interno della cellula tubulare.

SST = Somatostatina; SSTR = recettore della somatostatina; V2RA = antagonisti del recettore A2 della vasopressina; $A C-6=$ adenil-ciclasi $6 ;$ ER = reticolo endoplasmatico; $\mathrm{mTOR}=$ mammalian target della rapamicina; $\mathrm{PC} 1$ = policistina $1 ; \mathrm{PC2}=$ policistina $2 ; \mathrm{PKA}=$ protein-chinasi $\mathrm{A} ; \mathrm{TSC}$ = tuberina (TSC2) e amartina (TSC1): proteine coinvolte nella sclerosi tuberosa; $\mathrm{CAMP}=$ adenosina ciclica monofosfato; $\mathrm{CFTR}=$ cystic fibrosis transmembrane conductance regulator (proteina coinvolta nella fibrosi cistica).

progressivamente di numero e dimensioni, conducendo a un aumento volumetrico massiccio del rene e, inevitabilmente, alla perdita della funzione renale, che può condurre all'uremia terminale (ESRD) (4). Le cisti inizialmente si sviluppano come piccole dilatazioni dei tubuli renali a causa della mutazione in uno dei due alleli dei geni PKD1 o PKD2 (first hit); successivamente, si hanno l'inattivazione somatica dell'allele non mutato rimanente della policistina (second hit) e una terza fase rappresentata da un evento precipitante (p. es., lesioni ischemiche o sostanze nefrotossiche) in grado di stimolare la proliferazione cellulare e la crescita delle cisti (5).

L'espansione delle cisti, in seguito, è causata da una maggiore proliferazione delle cellule epiteliali, dalla continua secrezione di fluidi (Fig. 1), dal distacco dalla struttura tubulare originale, da anomalie della matrice extracellulare, ovviamente 
dall'espressione del gene alterato e dall'anomala polarità delle cellule, a cui si aggiungono infiammazione, aumento dell'apoptosi e altri fattori ambientali $(4,6,7)$. II meccanismo di formazione delle cisti si verifica anche a livello epatico, nel pancreas e nella milza; possono essere riscontrati aneurismi intracranici e difetti valvolari cardiaci (8).

Il trattamento dell'ADPKD è stato, finora, essenzialmente di supporto, atto a trattare o a ridurre la sintomatologia causata dalla malattia di base, che, ancora oggi, risulta incurabile se non con il trapianto (9); tuttavia, recenti scoperte sulla fisiopatologia della malattia hanno stimolato la ricerca a cercare nuovi bersagli terapeutici nel tentativo di arrestare la progressione dell'ADPKD $(10,11)$. Nuove terapie per il trattamento della patologia cistica, inizialmente sperimentate su modelli animali, sono state successivamente utilizzate sull'uomo con risultati che, in alcuni casi, sono stati incoraggianti.

\section{Nuove strategie terapeutiche sperimentali}

Fino a qualche anno addietro, la terapia del rene policistico era considerata solo di supporto. Negli ultimi 7 anni, sono stati pubblicati numerosi studi, che hanno fatto riconsiderare tale assunto, dando nuove speranze a chi puntava a modificare la storia naturale dell'ADPKD, facendo affacciare la ricerca a nuovi orizzonti in ambito farmacologico, anche se, apparentemente, ancora precoci.

\section{Ruolo della terapia antipertensiva}

Il ruolo della terapia antipertensiva nei pazienti affetti da ADPKD ha assunto nel tempo una rilevanza di estrema importanza. II razionale, però, di tale terapia era rivolto al trattamento di un sintomo causato dalla malattia policistica e non tanto alla modifica dell'evoluzione o della storia naturale della malattia stessa.

L'ipertensione arteriosa è una condizione molto comune nei soggetti affetti da ADPKD. Un ruolo patogenetico rilevante è giocato dalle cisti progressivamente crescenti che inducono l'ischemia locale e, conseguentemente, l'attivazione del sistema renina-angiotensina (RAAS). In questi pazienti, l'ipertensione induce l'insorgenza di ipertrofia ventricolare sinistra (LVH) e contribuisce al progressivo aumento del volume renale totale e il peggioramento della funzione renale. Dato il meccanismo fisiopatologico appena descritto in breve, i farmaci antipertensivi più idonei comprendono sostanzialmente due classi farmacologiche: gli inibitori dell'enzima di conversione (ACE-i) e/o gli antagonisti del recettore dell'angiotensina (ARBs) (12).

In uno studio di fase III, in doppio cieco controllato farmaco contro placebo (HALT-PKD [Studio A]; ClinicalTrials. gov Identifier: NCT00283686) (13), 558 pazienti ipertesi con diagnosi di ADPKD e con un eGFR $>60 \mathrm{~mL} / \mathrm{min}$ sono stati assegnati in modo randomizzato a ricevere o un ACE inibitore (lisinopril) più un bloccante del recettore dell'angiotensina (telmisartan) o lisinopril e placebo, con l'obiettivo di raggiungere o uno standard pressorio dato dai valori compresi fra $120 / 70$ e 130/80 mmHg o un obiettivo di bassa pressione arteriosa, con valori da 95/60 a 110/75 mmHg.

Gli Autori hanno osservato un aumento più lento del volume renale totale nel gruppo mantenuto al bersaglio più a bassa pressione arteriosa rispetto al gruppo con il target pressorio standard ( $P=0.006$ vs $P=0.52$ ). Al contrario, nessuna differenza significativa è stata riscontrata tra i due bracci di sperimentazione per quanto riguarda l'aumento di volume dei reni e, soprattutto, l'eGFR ( $P=0.55)$. Nel gruppo a basso target pressorio si sono registrate una diminuzione della LVH e una ridotta escrezione urinaria di albumina.

Lo studio B HALT-PKD (14) è uno studio-controllo di fase III in doppio cieco, in cui 486 pazienti con ADPKD presentanti un eGFR compreso tra i 25 e i $60 \mathrm{~mL} / \mathrm{min}$ hanno ricevuto in modo randomizzato lisinopril e placebo o lisinopril e telmisartan alle dosi necessarie per ottenere una pressione arteriosa tra 110/70 e 130/80 mmHg. Gli Autori hanno concluso che il trattamento con un ACE inibitore era in grado di controllare la pressione arteriosa in molti pazienti con IRC allo stadio III e che l'aggiunta di un bloccante del recettore ARBS non influenza il declino dell'eGFR.

\section{Inibitori del "Mammalian target of rapamycin", mTOR inhibitors}

II "Mammalian target of rapamycin" (mTOR) è una serin/ treonina chinasi che regola il metabolismo, la crescita, la proliferazione, la motilità e la sopravvivenza cellulari (15); essa controlla anche la traduzione di mRNA, la sintesi delle proteine e le funzioni mitocondriali (16) ed è fondamentale nella biologia delle cellule $\mathrm{T}$, anche se il ruolo di mTOR nella regolazione immunitaria deve essere definito ancora meglio (17). La via del mTOR sembra essere disregolata in diverse patologie umane, tra cui il diabete mellito, l'obesità e le patologie oncologiche (18).

Le evidenze succitate rappresentano il razionale per l'uso degli inibitori di mTOR (sirolimus, everolimus, ecc.) nel trattamento di alcune neoplasie umane in casi selezionati, come fase iniziale non-small cell lung cancer (NSCLC) (19), carcinoma renale avanzato (20), carcinoma mammario in stadio avanzato positivo ai recettori ormonali positivi e negativo per il recettore 2 del fattore di crescita epidermico umano (21) e sarcoma di Kaposi nei riceventi di trapianto renale (22) e nella prevenzione del rigetto di trapianto $(23,24)$.

mTOR esiste come parte di due distinti complessi proteici, chiamato mTOR complex 1 (mTORC1) e mTOR complex 2 (mTORC2). Entrambi sembrano essere impropriamente attivati in corso di ADPKD, come dimostrato in modelli sperimentali (25) e negli studi umani (26). Il coinvolgimento di un signalling interno mediato da mTOR nell'ADPKD è confermato dalla capacità della rapamicina, un macrolide batterio-derivato noto anche come sirolimus (27), di ridurre la crescita delle cisti e 
di migliorare la funzione renale, impedendo la proliferazione delle cellule epiteliali e riducendo la fibrosi e stimolando l'apoptosi delle cellule di rivestimento in un modello sperimentale murino (28). In un altro studio, il sirolimus inibisce il mTORC1 e la crescita delle cisti renali, ma non ha avuto effetti sul deterioramento della funzione renale nei topi con la mutazione del gene PKD2 (25).

Alcuni studi clinici sono stati eseguiti anche per testare l'efficacia dell'inibizione di mTOR, in soggetti affetti da ADPKD.

Lo studio "SIRENA" (ClinicalTrials.gov Identifier: NCT00491517) (29) è stato tra i primi studi a dimostrare che $i$ risultati ottenuti in modelli animali di ADPKD trattati con sirolimus potrebbero essere almeno parzialmente riprodotti negli esseri umani. Questo studio randomizzato di fase II è stato ideato per valutare gli effetti del sirolimus sulla progressione dell'ADPKD. Al gruppo sperimentale, composto da 21 pazienti con ADPKD e funzione renale normale o insufficienza renale da lieve a moderata, è stato somministrato il sirolimus (a partire da una dose giornaliera per via orale di $3 \mathrm{mg}$ regolata per raggiungere livelli ematici di sirolimus entro il range terapeutico di $10-15 \mathrm{ng} / \mathrm{mL}$, in seguito modificata per raggiungere $5-10 \mathrm{ng} / \mathrm{mL}$ ) come add-on alla terapia standard per 6 mesi; gli outcome presi in esame erano il volume totale del rene, il GFR, il volume delle cisti e il volume del parenchima renale. II sirolimus ha determinato, rispetto al trattamento standard, un rallentamento nella crescita delle cisti ed un aumento del parenchima renale totale. Inoltre, nel gruppo con sirolimus, si è registrato un aumento dell'albuminuria e della proteinuria.

Risultati simili sono stati ottenuti in uno studio di fase III di 18 mesi, randomizzato controllato, open-label (ClinicalTrials. gov Identifier: NCT00346918), in cui 100 pazienti adulti con ADPKD e con un'insufficienza renale lieve sono stati randomizzati a ricevere sirolimus ( $2 \mathrm{mg}$ al giorno) o una terapia convenzionale. Al termine del periodo di osservazione, il volume totale del rene e il GFR non differivano significativamente tra i due gruppi, mentre il tasso di escrezione urinaria di albumina è stato maggiore nei pazienti trattati con sirolimus (30).

Sembra che il metodo di valutazione della funzione renale sia rilevante per controllare il potenziale vantaggio fornito dal sirolimus nei pazienti con ADPKD.

Un più recente studio pilota di fase $\mathrm{I} / \mathrm{II}$, randomizzato, open-label (ClinicalTrials.gov Identifier: NCT00286156) (31) ha dimostrato che una bassa dose di terapia con sirolimus in 1 anno può migliorare il GFR ottenuto con I(125)-iotalamato rispetto al trattamento standard, ma non influenza il GFR stimato (eGFR), calcolato in base alla formula CKD-EPI, né il volume renale totale.

Inoltre, uno studio di sicurezza/efficacia randomizzato in singolo cieco, con assegnazione parallela, della durata di 2 anni, per il quale sono stati arruolati 16 pazienti ( 8 dei quali hanno ricevuto solo telmisartan, mentre i rimanenti 8 sono stati trattati con sirolimus più telmisartan), ha dimostrato che l'aggiunta di un inibitore di mTOR potrebbe ritardare l'aumento del volume renale totale durante i primi 6 mesi di tratta- mento, ma non arrestare tale crescita dopo tale periodo (32).

Un altro studio di fase II (studio RAPYD, EUDRACT N. 2007 006557-25) (33) ha messo in confronto tre gruppi sperimentali: ramipril, ramipril + rapamicina ad alte dosi e ramipril + basse dosi di rapamicina, in 55 pazienti con ADPKD di tipo I. Gli Autori hanno riportato un aumento del volume totale renale in tutti i gruppi dopo 2 anni di terapia, anche se tale incremento volumetrico era meno rilevante nei pazienti trattati con la dose rapamicina superiore rispetto agli altri due gruppi.

Problemi di sicurezza e di efficacia hanno determinato I'interruzione precoce dello studio "SIRENA II". Quest'ultimo era uno studio di fase II, randomizzato, open-label (ClinicalTrials.gov Identifier: NCT01223755), che mirava a valutare gli effetti del sirolimus sulle variazioni del GFR e sui parametri renali nonché sul volume del fegato, in pazienti con ADPKD e grave insufficienza renale.

Un altro studio (ClinicalTrials.gov Identifier: NCT00920309) è stato interrotto a causa della mancanza di beneficio nel trattamento di ADPKD con inibitori di mTOR, come riportato in due grandi trial pubblicati sul NEJM $(30,34)$. Quest'ultimo è uno studio in cui si sono testate l'efficacia e la sicurezza di un altro inibitore di mTOR, l'everolimus. In questo studio, 433 pazienti con ADPKD sono stati assegnati in modo casuale a ricevere placebo o everolimus e poi seguiti per 2 anni. Gli Autori hanno osservato che l'everolimus era in grado di rallentare l'aumento del volume renale totale, ma non il declino della funzione renale.

Una meta-analisi è stata condotta sui 4 studi più importanti randomizzati per valutare l'efficacia e la sicurezza del sirolimus nel trattamento dell'ADPKD, in modo da confrontarne possibili benefici e/o possibili controindicazioni (35). Nei gruppi sperimentali trattati con sirolimus, si registra sempre un volume renale totale minore rispetto ai rispettivi gruppi di controllo. La differenza media (MD) del volume renale totale post-trattamento rispetto al gruppo di controllo era di -234.74 ( $P=0.01)$. Tuttavia, il GFR non ha raggiunto una differenza statisticamente significativa tra i due gruppi. La differenza media standard (SMD) del GFR dopo la terapia era 0.24 (95\% confidence interval [Cl], 0.05 e $0.52 ; \mathrm{P}=0.11$ ). Inoltre, il sirolimus sembrava aumentare l'escrezione di proteine nelle urine $(P=0.002)$. Stomatite aftosa e faringiti sono riportati più comunemente nel gruppo terapia di sirolimus rispetto al gruppo di controllo $(P<0.000001)$.

In conclusione, gli inibitori di mTOR risultano sicuri e sembrano avere alcuni effetti nei pazienti con ADPKD, soprattutto nel ridurre l'incremento del volume renale, ma sembrano non avere alcun effetto sul rallentamento del declino del GFR e si associano a un aumento dell'escrezione urinaria di proteine. Per tale motivo, non risulta ancora possibile proporre questo trattamento nella pratica clinica e il ruolo terapeutico di questi farmaci rimane incerto. 


\section{Analoghi della somatostatina}

Come è noto, l'ADPKD è molto frequentemente associata ad altre condizioni patologiche specifiche, la più importante delle quali è, senza alcun dubbio, lo sviluppo di cisti nel contesto del parenchima epatico, condizione che configura la malattia policistica del fegato (PLD) associata all'ADPKD.

Sebbene molte caratteristiche nel controllo della crescita delle cellule epatiche e renali, rispettivamente nella PLD e nell'ADPKD, sembrino essere diverse, esistono evidenze biologiche per cui la formazione e la progressione delle cisti parenchimali apparirebbero simili nei due organi (36) e ciò costituisce la base per lo sviluppo di nuove terapie mirate nel controllo della proliferazione e nel progresso di entrambe le condizioni. Da un punto di vista epidemiologico, la PLD si verifica nel 7590\% dei pazienti con ADPKD. Esistono due forme di PLD: la PLD isolata, caratterizzata dalla presenza di cisti solo nel fegato, e la PLD associata all'ADPKD, in cui le cisti si sviluppano sia nel fegato che nel parenchima renale (37). Da un punto di vista eziopatogenetico, la forma isolata di PLD è dovuta a mutazioni nel SEC63 e a geni PRKCSH. I prodotti proteici dei geni SEC63 e PRKCSH (proteine sec63.protein ed epatocistina, rispettivamente) svolgono un ruolo importante nella trasformazione delle proteine e le mutazioni in questi geni influenzano proteine come policistina 1 e policistina 2, che, come abbiamo detto precedentemente, sono coinvolte nel trasporto di fluidi attraverso i vari compartimenti extracellulari e intracellulari e nella crescita di cellule epiteliali (38). Al contrario, la forma PLD ADPKD-associata è causata da mutazioni in entrambi i geni PKD1 o PKD2, come accade nel rene. Questo risultato è stato osservato anche in modelli murini specifici per l'ADPKD, portatori di queste mutazioni genetiche specifiche. Quei pazienti che soffrono di PLD e PKD tipicamente sviluppano insufficienza renale e richiedono la dialisi e/o il trapianto di rene, ma raramente hanno bisogno di un trapianto di fegato (39).

Recenti evidenze sperimentali suggeriscono che gli analoghi della somatostatina (SSA) potrebbero offrire qualche beneficio nel ridurre il processo citogenetico a carico dei reni (Fig. 1) e del fegato in corso di PLD associata ad ADPKD (40), così come nell'inibizione di mTOR (41) e del VEGF (42).

La somatostatina (SST) è un ormone sintetizzato a livello ipotalamico e trasportato alla ghiandola pituitaria anteriore e da cui viene successivamente dismesso. La SST agisce attraverso il reciproco riconoscimento di sei diversi recettori presenti sulla superficie delle cellule (SSTR), membri della famiglia dei recettori accoppiati alle proteine G. Comprendere la relazione fra struttura e funzione delle SST e dei loro recettori ha favorito lo sviluppo di una varietà di agenti farmacologici innovativi, come gli analoghi delle somatostatine (SSA) (43).

Alcuni studi si sono concentrati sull'efficacia di alcuni SSA, come lanreotide, octreotide o pasireotide nel trattamento dell'ADPKD, con il coinvolgimento renale ed extrarenale (fegato ed ovaio principalmente).
Lanreotide è un SSA sintetico che si lega agli stessi recettori della SST, ma, a differenza dell'ormone secreto naturalmente, l'analogo lega i recettori periferici con una maggiore affinità mantenendo un'attività simile (i cosiddetti "full agonists"). Tuttavia, mentre la SST si distribuisce rapidamente nel corpo, lanreotide ha un'emivita plasmatica molto duratura e produce effetti più prolungati (44).

Alcuni studi sono stati progettati per valutare se lanreotide potesse essere in grado di ridurre le cisti epatiche. Tra questi, i più rilevanti sono lo studio LOCKCYST I e LOCKCYST II, in cui veniva valutato il possibile trattamento con lanreotide in pazienti con PLD e ADPKD. In tali trial, sono state esplorate la sicurezza e l'efficacia di due diverse dosi di lanreotide (90 e $120 \mathrm{mg}$ ) per il trattamento della PLD. L'endpoint primario era quello di valutare la sicurezza e l'efficacia del farmaco nella riduzione del volume epatico. L'endpoint secondario era la variazione relativa di volume renale totale e cambiamenti del GFR. Nel primo studio RCT, dopo 6 mesi di trattamento, più del $50 \%$ dei pazienti ha mostrato una riduzione del volume epatico $>100 \mathrm{~mL}$, in particolar modo nel braccio sperimentale trattato con lanreotide a $120 \mathrm{mg}$. La popolazione dello studio era costituita da tre gruppi che hanno ricevuto ogni 4 settimane i seguenti trattamenti: placebo $(n=26)$, lanreotide $90 \mathrm{mg}$ ( $n=55$ ) o lanreotide $120 \mathrm{mg}(\mathrm{n}=51)$. L'osservazione principale di questo studio è stata che sia nel gruppo trattato con lanreotide $90 \mathrm{mg}$ che in quello trattato con lanreotide $120 \mathrm{mg}$ si registrava un decremento del volume epatico dopo 6 mesi e questo effetto è statisticamente più pronunciato quando viene utilizzata la dose più alta del farmaco. Dal momento che una dose inferiore si associa a minori effetti collaterali, in caso di intolleranza a una dose di $120 \mathrm{mg}$ è possibile ridurre la dose a $90 \mathrm{mg}$, che, comunque, mantiene un effetto benefico rispetto al placebo. Un altro elemento che è venuto fuori da questo studio è l'osservazione di una diminuzione del volume renale nei gruppi sperimentali. La riduzione del volume renale osservata nel gruppo sperimentale "lanreotide 120 mg" è risultata significativamente diversa rispetto al gruppo di controllo. Nel gruppo placebo, il volume renale è aumentato del $3 \%$ in 6 mesi, in linea con i tassi di crescita osservati in altri studi, elemento che non si è osservato nel gruppo sperimentale. La funzione renale è stata stimata per mezzo della formula CKD-EPI. I ricercatori non hanno osservato alcun effetto benefico significativo sulla velocità di filtrazione glomerulare, ma questo può essere dovuto alla durata dell'osservazione relativamente breve (6 mesi) (45).

Dopo il lanreotide, anche l'octreotide (Octreotide LAR), un altro analogo sintetico dell'ormone naturale SST, è stato oggetto di un'indagine come una possibile terapia in ADPKD. Gli studi preclinici nel ratto PCK (un modello di ADPKD associata a PLD) ha dimostrato come l'octreotide riduca i livelli di cAMP nel rene e nelle vie biliari e rallenti la progressione della cistogenesi epato-renale (46). Questa è una prova importante sui possibili effetti benefici dell'octreotide nella PLD associata ad ADPKD. 
In uno studio randomizzato, controllato con placebo, è stato indagato il profilo di rischio/beneficio del trattamento di 6 mesi con octreotide a lunga durata d'azione (40 mg per via intramuscolare ogni 28 giorni) rispetto al placebo, in pazienti con ADPKD, insufficienza renale lieve/moderata e nessuna evidenza di altre malattie renali. II volume del rene, valutato in TC multislice, in sei mesi è aumentato di $71 \pm 107 \mathrm{~mL}$ $(p<0.05)$ nel gruppo sperimentale trattato con octreotide e di $162 \pm 114 \mathrm{ml}(\mathrm{p}<0.01)$ nel gruppo placebo. II GFR non è cambiato significativamente nei due gruppi nel periodo di trattamento. Questi risultati hanno suggerito che una terapia con octreotide a breve termine ( 6 mesi) è sicura e può rallentare l'espansione del volume renale in pazienti con ADPKD (47). Un'analisi post hoc del precedente studio ha evidenziato come l'octreotide induca una significativa diminuzione del volume epatico da $1595 \pm 478 \mathrm{~mL}$ a $1524 \pm 453 \mathrm{~mL}$, volume che non è sensibilmente cambiato nel gruppo di controllo. Questa diminuzione del volume epatico è risultata significativamente correlata con la concomitante riduzione del volume renale $(r=0.67 ; p<0.05)$ nel gruppo trattato con octreotide, ma non nel controllo (48).

In un altro studio clinico (ClinicalTrials.gov Identifier: NCT00426153) in doppio cieco, randomizzato e controllato, è stato indagato il trattamento con octreotide long acting per più di 1 anno in pazienti con grave PLD dovuta a PLD autosomica dominante (ADPLD) o ad ADPKD. Come per gli studi LOCKCYST, l'endpoint primario era rappresentato dal cambiamento nel volume del fegato totale misurato con la risonanza magnetica (MRI); endpoint secondari erano variazioni di volume renale totale alla MRI e la funzione renale misurata con eGFR. Un totale di 42 pazienti è stato arruolato e randomizzato in questo studio, di cui 28 facenti parte del gruppo sperimentale con octreotide LAR e 14 nel gruppo di controllo. II gruppo sperimentale ha ricevuto octreotide LAR a $40 \mathrm{mg}$ ogni 28 giorni o alla dose massima tollerata ( 2 dei pazienti arruolati non hanno concluso la sperimentazione clinica). Dalle analisi combinate dei 26 pazienti che hanno ricevuto 1 anno di octreotide è stata osservata una riduzione complessiva del $6.08 \%$ del volume totale epatico, mentre, nei pazienti che sono stati randomizzati a placebo $(n=14)$, lo stesso è aumentato dello $0.9 \pm 8.35 \%$ rispetto al basale durante il primo anno dall'inizio della sperimentazione clinica. Inoltre, durante il primo anno di trattamento con octreotide, il volume renale totale non si è modificato rispetto alla baseline, a differenza delle modifiche volumetriche pari all' $8.61 \pm 10.07 \%$ nei pazienti del gruppo di controllo. Questi dati suggeriscono che la terapia con octreotide può anche avere un effetto benefico sulla crescita renale almeno per il primo anno di trattamento. Infatti, mentre l'incremento del volume renale sembra essere inibito durante il primo anno dal trattamento, questo sembra perdere di efficacia nel corso del secondo anno. Questo studio non ha rilevato effetti benefici dell'octreotide sulla funzione renale (49).

Un altro studio di estrema importanza nella comprensio- ne del possibile ruolo delle SST nel trattamento della malattia policistica è lo studio ALADIN, studio randomizzato controllato condotto per valutare l'efficacia dell'octreotide nell'ADPKD. In questo caso, l'obiettivo era di valutare l'effetto di 3 anni di trattamento con octreotide sulla crescita delle cisti renali e sul declino della funzione renale nei pazienti con ADPKD. I pazienti (con GFR $>40 \mathrm{~mL} / \mathrm{min}$ ) sono stati assegnati in modo casuale al trattamento con due iniezioni intramuscolari di octreotide $(20 \mathrm{mg}$ ) ( $\mathrm{n}=40$, gruppo sperimentale) o con soluzione di cloruro di sodio $0.9 \%$ ( $n=39$, gruppo di controllo ) ogni 28 giorni. L'endpoint primario era rappresentato dal cambiamento nel volume renale totale misurato alla MRI a uno e a tre anni di follow-up. Dopo un anno, l'incremento del volume renale si è mostrato significativamente inferiore $(p=$ 0.032) nel gruppo sperimentale rispetto al gruppo placebo. Al follow-up di tre anni, l'aumento medio del volume renale totale nel gruppo sperimentale era numericamente inferiore rispetto al gruppo placebo, ma non in modo significativo. Inoltre, sempre nel primo anno, il GFR è diminuito del $10.8 \%$ nel gruppo sperimentale e del $9.7 \%$ nel gruppo di controllo. Interessante è stato notare come i valori medi di GFR siano rimasti stabili nel gruppo sperimentale, mentre sono progressivamente diminuiti nel gruppo placebo, fino a 3 anni di follow-up. È importante sottolineare che la riduzione iniziale del GFR era controbilanciata da un più ridotto declino del GFR nelle fasi successive, coerentemente con l'ipotesi che effetto del trattamento potrebbe essere mediato da un miglioramento dell'iperfiltrazione glomerulare (50).

Se questi risultati suggeriscono che gli SSA come lanreotide e octreotide potrebbero essere la nuova strategia terapeutica nel trattamento della PLD associata (o meno) ad ADPKD, alcune evidenze ottenute da studi preclinici suggeriscono che ci potrebbero essere altri SSA più potenti, come la pasireotide (PAS), un SSA con alta affinità recettoriale. Uno studio di confronto tra octreotide e PAS ha mostrato che quest'ultima era più potente, del 30-45\%, nel ridurre i livelli di cAMP e, conseguentemente, la proliferazione delle cellule, riducendo la crescita delle cisti in vitro e inibendo la cistogenesi epatorenale in vivo nei modelli murini (51).

\section{Antagonisti del recettore della vasopressina V2 - tolvaptan}

Alcuni studi sperimentali in corso di ADPKD suggeriscono come la proliferazione delle cellule epiteliali delle cisti renali e l'accumulo di liquidi $\mathrm{Cl}^{-}$-dipendenti al loro interno siano promosse dall'ormone antidiuretico arginina vasopressina (AVP) e dal suo secondo messaggero intracellulare 3',5'-adenosina monofosfato ciclico (cAMP). Da un punto di vista fisiologico, l'AVP si lega ai recettori V2 sulla superficie delle cellule del nefrone distale e dei dotti collettori che rappresentano le principali strutture da cui si sviluppano le cisti. L'interazione di AVP con i recettori V2, sovraespressi e iperattivati nelle cellule epiteliali cistiche, porta a un au- 
mento della produzione di cAMP intracellulare che è, a sua volta, responsabile della crescita della cisti, stimolando sia la proliferazione delle cellule epiteliali sia la secrezione transepiteliale di $\mathrm{Cl}$, quest'ultimo in grado di guidare per osmosi il flusso dell'acqua nella cavità cistica (Fig. 1) (1, 52, 53). La rilevanza del pathway molecolare dell'AVP nella patogenesi dell'ADPKD è dimostrata dal ridotto sviluppo di cisti e da una funzione renale conservata osservati in modelli animali attraverso meccanismi come la soppressione del rilascio di AVP dopo l'assunzione di acqua ad alte dosi (54), la soppressione del gene AVP (55) e l'antagonismo del recettore V2 (56-58).

L'identificazione di questo meccanismo ha spinto la ricerca a ipotizzare un ruolo terapeutico per antagonisti del recettore V della vasopressina, noti anche come "agenti acquaretici", nel trattamento di pazienti con ADPKD. In particolare, gli studi clinici si stanno concentrando sull'antagonista del recettore V2 chiamato tolvaptan. Le attuali indicazioni terapeutiche per I'uso degli acquaretici nella pratica clinica sono la Sindrome da inappropriata secrezione di ormone antidiuretico (SIADH), I'insufficienza cardiaca congestizia e la cirrosi epatica $(59,60)$.

Uno studio di fase II, randomizzato, in assegnazione parallela, open-label (ClinicalTrials.gov Identifier: NCT00413777) è stato condotto per valutare il profilo di sicurezza a lungo termine del tolvaptan (alle dosi di $30-120 \mathrm{mg} /$ die) e poi per stabilire la dose massima tollerata, in 46 soggetti affetti da ADPKD; gli outcome secondari includevano cambiamenti nell'osmolalità urinaria, il volume renale totale e il GFR stimato (eGFR). L'indagine, completata nel Giugno del 2010, ha permesso di acquisire alcuni dati di efficacia e, successivamente, lo stesso gruppo di pazienti è stato arruolato insieme a un gruppo di ulteriori 17 soggetti già inclusi in uno studio preliminare di fase II (ClinicalTrials.gov Identifier: NCT00841568) per valutare la sicurezza a lungo termine e l'efficacia dell'OPC-41061 (codice di designazione del tolvaptan). Secondo i risultati ottenuti dallo studio, il tolvaptan è stato in grado di rallentare la crescita di cisti, ma tutti i partecipanti che hanno completato 3 anni di trattamento con tolvaptan (51 soggetti, pari al 81\%) hanno presentato eventi avversi (61).

II più grande e importante studio designato per testare l'impatto del tolvaptan sulla progressione dell'ADPKD è il "TEMPO 3:4 Trial", uno studio di fase III, multicentrico, in doppio cieco, controllato con placebo e della durata di 3 anni (ClinicalTrials.gov Identifier: NCT00428948) (62). Complessivamente, 1445 pazienti adulti affetti da ADPKD, con un volume renale totale pari o superiore a $750 \mathrm{~mL}$ e un eGFR $>60 \mathrm{~mL} / \mathrm{min}$, sono stati randomizzati in un rapporto $2: 1$ per ricevere il tolvaptan (dose giornaliera totale compresa tra 60 e $120 \mathrm{mg}$, in base alla tollerabilità riferita dal paziente) o il placebo. L'outcome primario era rappresentato dal tasso di variazione percentuale del volume totale del rene. Come endpoint secondari, gli Autori hanno selezionato un outcome composito, costituito dal tempo della progressione clinica (rivelato da peggioramento dei marker di funzionalità renale, presenza di dolore renale, ipertensione arteriosa ed escrezio- ne urinaria di albumina) e dalla velocità di progressione della funzione renale (eGFR).

Una riduzione statisticamente significativa dell'incremento del volume renale totale è stata riportata nel gruppo di pazienti trattati con tolvaptan rispetto ai controlli. Anche il declino della funzione renale si è mostrato più lento nel gruppo trattato. Un'elevata incidenza di effetti avversi è stata registrata sia nel gruppo tolvaptan (97.9\%) che nel gruppo placebo (97.1\%); tali eventi sono stati legati soprattutto a un aumento dell'acquaresi (sete, poliuria, nicturia e polidipsia) nei pazienti trattati con tolvaptan, mentre, nei pazienti trattati con placebo, gli eventi avversi erano principalmente legati alla malattia di base, I'ADPKD (per esempio, dolore renale e mal di schiena, ematuria e infezioni del tratto urinario). Inoltre, il tolvaptan ha indotto un aumento dei livelli sierici di acido urico, del sodio e degli enzimi epatici in alcuni partecipanti (questi ultimi reversibili alla sospensione del farmaco). Tali eventi avversi hanno sollevato la questione circa la sicurezza e sottolineano l'importanza di monitorare questi parametri durante la terapia con tolvaptan.

In un'analisi "post-hoc", è stata indagata la differenza in termini di benefici terapeutici riscontrati nei gruppi sperimentali del TEMPO 3:4 Trial, stratificati in base allo stadio di insufficienza renale riscontrata durante l'arruolamento.

In sintesi, questa analisi post-hoc dello studio TEMPO 3:4 suggerisce che l'effetto inibitorio del tolvaptan sul tasso di crescita del rene è simile in pazienti con ADPKD attraverso gli stadi CKD1, CKD2 e CKD3, mentre l'effetto benefico sul declino della funzione renale, rilevato tramite l'eGFR, è più facilmente dimostrabile in pazienti con CKD2 o CKD3. Questa analisi mostra anche che questi effetti benefici sono raggiunti senza un aumento della frequenza di eventi avversi nelle fasi più avanzate della malattia, a eccezione di un aumento della frequenza di ipernatremia nello stadio CKD3. Dal momento che questa è un'analisi post-hoc, le suddette conclusioni devono essere interpretate comunque con cautela (63).

II gruppo di Boertien et al. (ClinicalTrials.gov Identifier: NCT01336972) (64) ha studiato gli effetti emodinamici a breve termine del tolvaptan in 29 soggetti con ADPKD, sia con normale funzione renale sia con insufficienza renale da lieve o moderata sia con grave compromissione della funzionalità renale. II GFR e il flusso plasmatico renale effettivo sono stati misurati tramite la clearance con I(125)-iotalamato e tramite la clearance I(131)-ippurico, rispettivamente. Gli Autori non hanno dimostrato alcuna influenza del tolvaptan sul profilo emodinamico renale. Inoltre, i pazienti con normale funzione renale o insufficienza renale lieve/moderata hanno mostrato una significativa riduzione del GFR, che è risultata reversibile dopo la sospensione del farmaco; tale diminuzione non è risultata statisticamente significativa nei pazienti con eGFR $<30 \mathrm{~mL} / \mathrm{min}$.

In conclusione, il tolvaptan è attualmente considerato come un farmaco altamente promettente per il trattamento dei pazienti con ADPKD. 
In precedenza, il tolvaptan era stato approvato in Giappone $(7.5,15$ e $30 \mathrm{mg}$ ) per l'indicazione aggiuntiva di ADPKD, in particolare per il controllo della progressione della malattia nei pazienti che già presentavano un aumento del volume del rene e il cui tasso di incremento volumetrico risultava essere in rapida crescita. Sulla base delle prove cumulative e, in particolare, dei risultati dello studio TEMPO 3:4 (62), il 26 Febbraio 2015, l'Agenzia europea per i medicinali (EMA) ha concesso l'approvazione per la commercializzazione del tolvaptan per i pazienti adulti con rapida progressione della malattia e con declino della funzione renale lieve/moderata (CKD1-3). Al momento, le autorità regolatorie in Giappone, Canada, Germania, Inghilterra, Scozia, Lussemburgo, Danimarca e Norvegia hanno autorizzato l'immissione in commercio di tolvaptan $\left(\operatorname{Jinarc}^{\circledR}\right)$, mentre, negli USA, la FDA ha richiesto, nel 2014, ulteriori chiarimenti in termini di efficacia e sicurezza. Nel Marzo 2016 l'autorità regolatoria italiana per il farmaco (AIFA) ha approvato l'uso del tolvaptan, ma ponendolo a carico del paziente (fascia $\mathrm{C}$ ).

Nel corso di quet'anno l'ERA-EDTA Working Groups of Inherited Kidney Disorders e l'European Renal Best Practice hanno deciso di preparare delle Linee Guida per aiutare il clinico nel decidere quando trattare i pazienti affetti da ADPKD con tolvaptan (65).

\section{Conclusioni}

Fino a oggi la gestione farmacologica del paziente con ADPKD si è basata su terapie di supporto che mirano a controllare la sintomatologia scatenata dalla malattia di base, l'ipertensione, e sul trattamento delle complicanze della malattia. Negli ultimi anni, importanti progressi sono stati fatti nella comprensione dei meccanismi fisiopatologici dell'ADPKD. Di conseguenza, numerosi trial si sono sviluppati nel tentativo di controllare la progressione della malattia e, conseguentemente, di cambiarne la storia naturale (66). Sebbene, per alcuni trattamenti sperimentali, ancora oggi manchi una forte evidenza scientifica che spinga al loro impiego clinico, al contrario, per altri farmaci, gli effetti terapeutici sono stati definiti meglio e, soprattutto, tra questi il tolvaptan sembra essere l'approccio terapeutico più promettente per la gestione dell'ADPKD.

Gli effetti degli inibitori di mTOR, sirolimus ed everolimus, sembrano essere controversi. Gli studi clinici effettuati per testare la loro efficacia hanno dimostrato che questi farmaci esercitano alcuni effetti nell'ADPKD; in particolar modo sono capaci di ridurre il volume renale e di rallentarne la crescita, ma la loro reale utilità terapeutica rimane incerta.

Attualmente, la terapia più promettente per il trattamento dell'ADPKD progressiva sembra essere il tolvaptan, un antagonista del recettore $\mathrm{V} 2$. I risultati più significativi su questo farmaco sono emersi dallo studio TEMPO 3:4, un trial clinico di grandi dimensioni che ha arruolato 1445 pazienti adulti affetti da ADPKD (62). I principali farmaci candidati al tratta- mento della crescita delle cisti epatiche sono gli analoghi della Somatostatina (SSA), come lanreotide (49), octreotide (50) e, recentemente, pasireotide (51). Questi farmaci sembrano essere di beneficio nei pazienti con PLD associata ad ADPKD, dal momento che sono in grado di diminuire la crescita delle cisti a livello epatico e, probabilmente, di avere anche alcuni effetti sul volume renale totale. A oggi, lanreotide e octreotide sembrano essere più promettenti nel ridurre il numero e il volume delle cisti renali ed epatiche e, soprattutto, nella stabilizzazione del GFR.

In conclusione, anche se alcuni risultati favorevoli con i nuovi schemi terapeutici sono stati già raggiunti, i dati disponibili a oggi non sono ancora sufficienti per stabilire se questi approcci possono fornire benefici consistenti nel rallentare la progressione dell'ADPKD nel prossimo futuro. È opinione dalla comunità scientifica che dovrebbero essere progettati ulteriori trial clinici randomizzati numericamente più grandi e di maggiore durata, in modo da confermare quanto riportato negli studi clinici pilota; bisogna anche aspettare l'immissione nel mercato per definire in maniera reale l'efficacia e la sicurezza delle terapie di cui ancora poco è conosciuto.

\section{Disclosures}

Financial support: No financial support was received for this submission. Conflict of interest: The authors have no conflict of interest.

\section{Bibliografia}

1. Reif GA, Yamaguchi T, Nivens E, et al. Tolvaptan inhibits ERKdependent cell proliferation, $\mathrm{Cl}+$ secretion, and in vitro cyst growth of human ADPKD cells stimulated by vasopressin. Am J Physiol Renal Physiol. 2011;301(5):F1005-13.

2. Trujillano D, Bullich G, Ossowski S, et al. Diagnosis of autosomal dominant polycystic kidney disease using efficient PKD1 and PKD2 targeted nextgeneration sequencing. Mol Genet Genomic Med. 2014;2(5):412-21.

3. Huang L, Lipschutz JH. Cilia and polycystic kidney disease, kith and kin. Birth Defects Res C Embryo Today. 2014;102(2):174-85.

4. Paul BM, Vanden Heuvel GB. Kidney: polycystic kidney disease. Wiley Interdiscip Rev Dev Biol. 2014;3(6):465-87.

5. Weimbs T. Third-hit signaling in renal cyst formation. J Am Soc Nephrol. 2011;22(5):793-5.

6. Bellinghieri G, Magaudda L, Santoro D, et al. Extracellular matrix abnormality may be responsible for cyst development. Contrib Nephrol. 1997;122:38-44.

7. Igarashi P, Somlo S. Genetics and pathogenesis of polycystic kidney disease. J Am Soc Nephrol. 2002;13(9):2384-98.

8. Mao Z, Xie G, Ong AC. Metabolic abnormalities in autosomal dominant polycystic kidney disease. Nephrol Dial Transplant. 2015;30(2):197-203.

9. LaRiviere WB, Irazabal MV, Torres VE. Novel therapeutic approaches to autosomal dominant polycystic kidney disease. Transl Res. 2015;165(4):488-98.

10. Torra R. Treatment of autosomal dominant polycystic kidney disease. Med Clin (Barc). 2014;142(2):73-9.

11. Cernaro V, Trifiro G, Lorenzano G, et al. New therapeutic strategies under development to halt the progression of renal failure. Expert Opin Investig Drugs. 2014;23(5):693-709.

12. Tesar V, Reiterova J. Hypertension in patients with polycystic kidney disease - incidence, pathogenesis, prognosis, therapy. 
Vnitr Lek. 2013;59(6):496-500.

13. Schrier RW, Abebe KZ, Perrone RD, et al. Blood pressure in early autosomal dominant polycystic kidney disease. N Engl J Med. 2014;371(24):2255-66.

14. Torres VE, Abebe KZ, Chapman AB, et al. Angiotensin blockade in late autosomal dominant polycystic kidney disease. N Engl J Med. 2014;371(24):2267-76.

15. Kim YC, Guan KL. mTOR: a pharmacologic target for autophagy regulation. J Clin Invest. 2015;125(1):25-32.

16. Morita M, Gravel SP, Hulea L, et al. mTOR coordinates protein synthesis, mitochondrial activity and proliferation. Cell Cycle. 2015;14(4):473-80.

17. Pollizzi KN, Powell JD. Regulation of $T$ cells by mTOR: the known knowns and the known unknowns. Trends Immunol. 2015;36(1):13-20.

18. Albert V, Hall MN. mTOR signaling in cellular and organismal energetics. Curr Opin Cell Biol. 2014;33C:55-66.

19. Owonikoko TK, Ramalingam SS, Miller DL, et al. A translational, pharmacodynamic, and pharmacokinetic phase IB clinical study of everolimus in resectable non-small cell lung cancer. Clin Cancer Res. 2015;21(8):1859-68.

20. Chiarini F, Evangelisti C, McCubrey JA, Martelli AM. Current treatment strategies for inhibiting $\mathrm{mTOR}$ in cancer. Trends Pharmacol Sci. 2015;36(2):124-35.

21. Chia S, Gandhi S, Joy AA, et al. Novel agents and associated toxicities of inhibitors of the pi3k/Akt/mtor pathway for the treatment of breast cancer. Curr Oncol. 2015;22(1):33-48.

22. Stallone G, Schena A, Infante B, et al. Sirolimus for Kaposi's sarcoma in renal-transplant recipients. N Engl J Med. 2005; 352(13):1317-23.

23. Caletti C, Granata S, Tomei P, et al. Extra-renal adverse effects of Mtor inhibitors: know them to optimize their use in renal transplantation. G Ital Nefrol. 2014;31:4.

24. Ganschow R, Pollok JM, Jankofsky M, Junge $G$. The role of everolimus in liver transplantation. Clin Exp Gastroenterol. 2014;7:329-43.

25. Zafar I, Ravichandran K, Belibi FA, et al. Sirolimus attenuates disease progression in an orthologous mouse model of human autosomal dominant polycystic kidney disease. Kidney Int. 2010;78 (8):754-61.

26. Shillingford JM, Murcia NS, Larson $\mathrm{CH}$, et al. The mTOR pathway is regulated by polycystin-1, and its inhibition reverses renal cystogenesis in polycystic kidney disease. Proc Natl Acad Sci USA. 2006;103(14):5466-71.

27. Amedei A, D'Elios MM. New therapeutic approaches by using microorganism-derived compounds. Curr Med Chem. 2012;19(22):3822-40.

28. Shillingford JM, Piontek KB, Germino GG, Weimbs T. Rapamycin ameliorates PKD resulting from conditional inactivation of Pkd1. J Am Soc Nephrol. 2010;21(3):489-97.

29. Perico N, Antiga L, Caroli A, et al. Sirolimus therapy to halt the progression of ADPKD. J Am Soc Nephrol. 2010;21(6): 1031-40.

30. Serra AL, Poster $D$, Kistler $A D$, et al. Sirolimus and kidney growth in autosomal dominant polycystic kidney disease. $\mathrm{N}$ Engl J Med. 2010;363(9):820-9.

31. Braun WE, Schold JD, Stephany BR, et al. Low-dose rapamycin (sirolimus) effects in autosomal dominant polycystic kidney disease: an open-label randomized controlled pilot study. Clin J Am Soc Nephrol. 2014;9(5):881-8.

32. Soliman A, Zamil S, Lotfy A, Ismail E. Sirolimus produced S-shaped effect on adult polycystic kidneys after 2-year treatment. Transplant Proc. 2012;44(10):2936-9.

33. Stallone G, Infante B, Grandaliano G, et al. Rapamycin for treatment of type I autosomal dominant polycystic kidney disease (RAPYD-study): a randomized, controlled study. Nephrol Dial Transplant. 2012;27(9):3560-7.

34. Walz G, Budde K, Mannaa M, et al. Everolimus in patients with autosomal dominant polycystic kidney disease. N Engl J Med. 2010;363(9):830-40.

35. Liu YM, Shao YQ, He Q. Sirolimus for treatment of autosomaldominant polycystic kidney disease: a meta-analysis of randomized controlled trials. Transplant Proc. 2014;46(1):66-74.

36. Strazzabosco M, Somlo S. Polycystic liver diseases: congenital disorders of cholangiocyte signaling. Gastroenterology. 2011; 140(7):1855-9.

37. Everson GT. Polycystic liver disease. Gastroenterol Hepatol. 2008;4(3):179-81.

38. Davila S, Furu L, Gharavi AG, et al. Mutations in SEC63 cause autosomal dominant polycystic liver disease. Nat Genet. 2004;36(6):575-7.

39. Kurbegovic $\mathrm{A}$, Cote $\mathrm{O}$, Couillard $\mathrm{M}$, et al. Pkd1 transgenic mice: adult model of polycystic kidney disease with extrarenal and renal phenotypes. Hum Mol Genet. 2010;19(7):1174-89.

40. Chrispijn M, Gevers TJ, Hol JC, et al. Everolimus does not further reduce polycystic liver volume when added to long acting octreotide: results from a randomized controlled trial. J Hepatol. 2013;59(1):153-9.

41. Spirli C, Okolicsanyi S, Fiorotto $R$, et al. Mammalian target of rapamycin regulates vascular endothelial growth factor dependent liver cyst growth in polycystin-2-defective mice. Hepatology. 2010;51(5):1778-88.

42. Spirli C, Okolicsanyi S, Fiorotto R, et al. ERK1/2-dependent vascular endothelial growth factor signaling sustains cyst growth in polycystin-2 defective mice. Gastroenterology. 2010;138(1):360-371.e7.

43. Barnett P. Somatostatin and somatostatin receptor physiology. Endocrine. 2003;20(3):255-64.

44. Rens-Domiano S, Reisine T. Biochemical and functional properties of somatostatin receptors. J Neurochem. 1992; 58(6):1987-96.

45. Temmerman F, Gevers T, Ho TA, et al. Safety and efficacy of different lanreotide doses in the treatment of polycystic liver disease: pooled analysis of individual patient data. Aliment Pharmacol Ther. 2013;38(4):397-406.

46. Masyuk TV, Masyuk Al, Torres VE, et al. Octreotide inhibits hepatic cystogenesis in a rodent model of polycystic liver disease by reducing cholangiocyte adenosine $3^{\prime}, 5^{\prime}$-cyclic monophosphate. Gastroenterology. 2007;132(3):1104-16.

47. Ruggenenti $P$, Remuzzi $A$, Ondei $P$, et al. Safety and efficacy of long-acting somatostatin treatment in autosomaldominant polycystic kidney disease. Kidney Int. 2005;68(1):206-16.

48. Caroli A, Antiga L, Cafaro M, et al. Reducing polycystic liver volume in ADPKD: effects of somatostatin analogue octreotide. Clin J Am Soc Nephrol. 2010;5(5):783-9.

49. Hogan MC, Masyuk TV, Page L, et al. Somatostatin analog therapy for severe polycystic liver disease: results after 2 years. Nephrol Dial Transplant. 2012;27(9):3532-9.

50. Caroli A, Perico N, Perna A, et al. Effect of long-acting somatostatin analogue on kidney and cyst growth in autosomal dominant polycystic kidney disease (ALADIN): a randomised, placebocontrolled, multicentre trial. Lancet. 2013;382(9903): 1485-95.

51. Masyuk TV, Radtke BN, Stroope AJ, et al. Pasireotide is more effective than octreotide in reducing hepatorenal cystogenesis in rodents with polycystic kidney and liver diseases. Hepatology. 2013;58(1):409-21.

52. Belibi FA, Reif G, Wallace DP, et al. Cyclic AMP promotes growth and secretion in human polycystic kidney epithelial cells. Kidney Int. 2004;66(3):964-73. 
53. Wallace DP. Cyclic AMP-mediated cyst expansion. Biochim Biophys Acta. 2011;1812(10):1291-300.

54. Nagao S, Nishii K, Katsuyama M, et al. Increased water intake decreases progression of polycystic kidney disease in the PCK rat. J Am Soc Nephrol. 2006;17(8):2220-7.

55. Wang $\mathrm{X}, \mathrm{Wu} \mathrm{Y}, \mathrm{Ward} \mathrm{CJ}$, et al. Vasopressin directly regulates cyst growth in polycystic kidney disease. J Am Soc Nephrol. 2008;19(1):102-8.

56. Gattone VH 2nd, Maser RL, Tian C, et al. Developmental expression of urine concentration-associated genes and their altered expression in murine infantiletype polycystic kidney disease. Dev Genet. 1999;24(3-4):309-18.

57. Gattone VH 2nd, Wang X, Harris PC, Torres VE. Inhibition of renal cystic disease development and progression by a vasopressin V2 receptor antagonist. Nat Med. 2003;9(10):1323-6.

58. Torres VE, Wang $X$, Qian $Q$, et al. Effective treatment of an orthologous model of autosomal dominant polycystic kidney disease. Nat Med. 2004;10(4):363-4.

59. Donato $\mathrm{V}$, Lacquaniti $\mathrm{A}$, Cernaro $\mathrm{V}$, et al. From water to aquaretics: a legendary route. Cell Physiol Biochem. 2014; 33(5):1369-88.

60. Cernaro V, Santoro D, Lacquaniti A, et al. From hyponatremia to tolvaptan. G Ital Nefrol. 2014;31:1.
61. Higashihara $E$, Torres VE, Chapman AB, et al. Tolvaptan in autosomal dominant polycystic kidney disease: three years' experience. Clin J Am Soc Nephrol. 2011;6(10):2499-507.

62. Torres VE, Chapman AB, Devuyst $O$, et al. Tolvaptan in patients with autosomal dominant polycystic kidney disease. N Engl J Med. 2012;367(25):2407-18.

63. Torres VE, Higashihara E, Devuyst O, et al. TEMPO 3:4 Trial Investigators. Effect of Tolvaptan in Autosomal Dominant Polycystic Kidney Disease by CKD Stage: Results from the TEMPO 3:4 Trial. Clin J Am Soc Nephrol. 2016.

64. Boertien $W E$, Meijer $E$, de Jong $P E$, et al. Short-term renal hemodynamic effects of tolvaptan in subjects with autosomal dominant polycystic kidney disease at various stages of chronic kidney disease. Kidney Int. 2013;84(6):1278-86.

65. Gansevoort RT, Arici M, Benzing T, et al. Recommendations for the use of tolvaptan in autosomal dominant polycystic kidney disease: a position statement on behalf of the ERA-EDTA Working Groups on Inherited Kidney Disorders and European Renal Best Practice. Nephrol Dial Transplant. 2016;31(3):337-48.

66. Santoro D, Pellicanò V, Visconti L, Trifirò G, Buemi M, Cernaro V. An overview of experimental and early investigational therapies for the treatment of polycystic kidney disease. Expert Opin Investig Drugs. 2015;24(9):1199-218. 